\title{
Nonequilibrium Molecular Dynamics Investigation of the Reduction in Friction and Wear by Carbon Nanoparticles Between Iron Surfaces
}

\author{
James P. Ewen ${ }^{1}$ (D) Chiara Gattinoni ${ }^{1} \cdot$ Foram M. Thakkar $^{2} \cdot$ Neal Morgan $^{3}$ • \\ Hugh A. Spikes ${ }^{1} \cdot$ Daniele Dini $^{1}$
}

Received: 27 May 2016/Accepted: 22 July 2016/Published online: 3 August 2016

(c) The Author(s) 2016. This article is published with open access at Springerlink.com

\begin{abstract}
For the successful development and application of novel lubricant additives, a full understanding of their tribological behaviour at the nanoscale is required, but this can be difficult to obtain experimentally. In this study, nonequilibrium molecular dynamics simulations are used to examine the friction and wear reduction mechanisms of promising carbon nanoparticle friction modifier additives. Specifically, the friction and wear behaviour of carbon nanodiamonds (CNDs) and carbon nano-onions (CNOs) confined between $\alpha$-iron slabs is probed at a range of coverages, pressures, and sliding velocities. At high coverage and low pressure, the nanoparticles do not indent into the $\alpha$-iron slabs during sliding, leading to zero wear and a low friction coefficient. At low coverage and high pressure, the nanoparticles indent into, and plough through the slabs during sliding, leading to atomic-scale wear and a much higher friction coefficient. This contribution to the friction coefficient is well predicted by an expression developed for macroscopic indentation by Bowden and Tabor. Even at the highest pressures and lowest coverages simulated, both nanoparticles were able to maintain separation of the opposing slabs and reduce friction by approximately $75 \%$ compared to when no nanoparticle was present, which
\end{abstract}

James P. Ewen

j.ewen14@imperial.ac.uk

1 Department of Mechanical Engineering, Imperial College London, South Kensington Campus, Exhibition Road, London SW7 2AZ, UK

2 Shell India Markets Private Limited, 8B RMZ Centennial Building, Kundanahalli Main Road, Bangalore, Karnataka 560048, India

3 Shell Global Solutions UK Ltd, Brabazon House, Manchester M22 0RR, UK agrees well with experimental observations. CNO nanoparticles yielded a lower indentation (wear) depth and lower friction coefficients at equal coverage and pressure with respect to CND, making them more attractive friction modifier additives. Potential changes in behaviour on harder and softer surfaces are also discussed, together with the implications that these results have in terms of the application of the studied nanoparticles as lubricants additives.

Keywords Molecular dynamics - Carbon nanoparticle . Nano-onion $\cdot$ Nanodiamond $\cdot$ Nanotechnology

\section{Introduction}

The need for greater energy efficiency in tribological systems has led to reduction in lubricant viscosity in order to reduce hydrodynamic losses; however, this means that an increasing number of engineering components operate in boundary lubrication conditions. As a result, lubricant additives that reduce friction and wear in boundary conditions are of increasing importance [1]. Recently, many types of metallic, inorganic, and carbon-based nanoparticles have attracted interest for use as boundary lubricant additives; a summary of these can be found in Ref. [1]. Carbon-based nanoparticles are particularly interesting since they have a high degree of structural stability and are chemically unreactive. Moreover, they do not contain elements such as sulphur, phosphorus, and certain metals which can be environmentally harmful and also poison engine exhaust after-treatment devices [1].

Since the discovery of Buckminster Fullerene $\left(\mathrm{C}_{60}\right)$, tribologists have been enthused about the possibility of utilising spherical molecules as 'nanoscale ball bearings' to 
reduce friction and wear [2]. Although $\mathrm{C}_{60}$ fullerene additives have been shown to reduce friction and wear under specific conditions [3-6], their action as molecular ball bearings has been widely disputed $[7,8]$ and their overall performance as potential lubricant additives has been somewhat disappointing $[1,7,8]$. However, larger carbon nanoparticle additives have shown more promise with regard to boundary friction and wear reduction, specifically carbon nanodiamonds (CND) and carbon nanoonions (CNO) [1]. CNDs can be formed from detonation experiments which yield extremely smooth, spherical particles with a diameter between 1 and $20 \mathrm{~nm}$ [9]. CNDs contain predominantly $s p^{3}$ carbons and have a mainly polycrystalline diamond structure. CNOs consist of nested icosahedral fullerenes, with each layer containing $60 \mathrm{n}^{2}$ carbon atoms, where $\mathrm{n}$ is an integer [10]. They can be formed by electron beam irradiation of amorphous carbon [10] as well as annealing of CNDs [11], which results in progressive graphitisation $\left(s p^{3}\right.$ to $\left.s p^{2}\right)$ from the surface to the core of the particle.

Both CND [9, 12-17] and CNO [18-26] have been shown to significantly reduce friction and wear of steel surfaces under boundary lubrication conditions. Specifically, CNO has been shown to reduce the friction coefficient by $\approx 85 \%$ when used as a solid lubricant [22]. Friction coefficient reductions of $\approx 50 \%$ have also been observed compared to a pure base oil when CNO [23] and CND [12] were used as additives. Under high-pressure boundary lubrication conditions, much of the base oil is squeezed out from between asperities [27, 28], leading to contact of opposing surfaces and high friction and wear. Large friction and wear benefits can still be observed when $\mathrm{CNO}$ and CND are used as additives, suggesting that they are able to reduce the contact of asperities more effectively than the base oil alone [12]. In comparative studies, CNO additives have yielded both lower [19, 23] and higher [17] friction and wear than CND additives, though the reasons for this disparity are, prior to this study, yet to be elucidated.

Despite considerable research, there is still much uncertainty as to the friction reduction mechanisms of carbon-based, and indeed most, nanoparticle friction modifier additives [1]. Many different mechanisms have been proposed including (a) sliding, (b) rolling, (c) exfoliation, and (d) polishing, as shown in Fig. 1.

Nanoparticles which are able to maintain separation of surface asperities will yield large reductions in friction and wear under boundary conditions [1]. This requires nanoparticles to be hard enough not to be plastically deformed under the high uniaxial pressures (GPa) experienced during boundary lubrication. However, if the nanoparticles are too hard, they may significantly indent into the surfaces, to the point that they no longer separate (a)
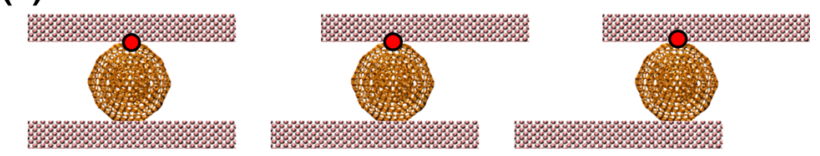

(b)
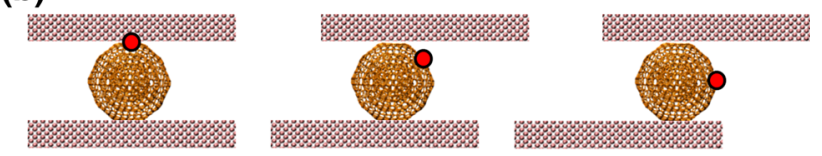

(c)
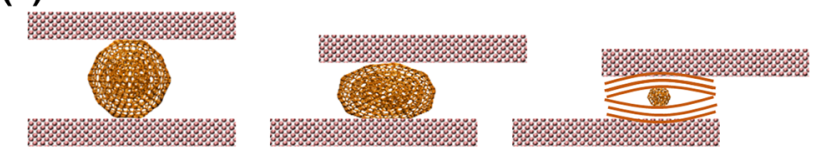

(d)
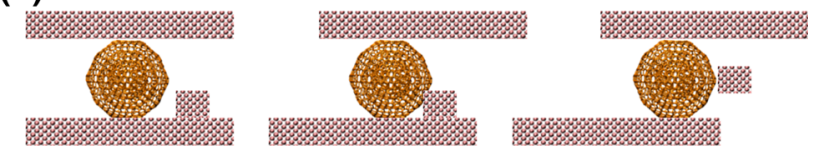

Fig. 1 Schematic showing the potential mechanisms of friction reduction by the presence of carbon nanoparticles pressed between sliding metal surfaces, adapted from Refs. [5] and [29]. a Sliding, b rolling, c exfoliation, and d polishing. Note that maintaining the separation of opposing surface asperities is likely to significantly reduce friction and wear in the boundary lubrication regime

asperities. Assuming that nanoparticles are able to prevent asperity contact, there is then the possibility of them rolling (Fig. 1a) or sliding (Fig. 1b) between the surfaces in order to reduce friction. It is unclear whether nanoparticles are able to act as nanoscale ball bearings, i.e. if nanoparticle rolling resistance reduces energy losses relative to sliding friction, as is the case for most macroscopic systems [7, 8]. Hard nanoparticles may also reduce friction through polishing (Fig. 1d), whereby asperities are smoothed by abrasion by the nanoparticles to reduce roughness and shift the system from boundary, towards mixed lubrication conditions [5]. Whilst this may initially provide a large benefit, it will quickly diminish in the presence of other running-in and smoothing processes. Moreover, the potential for abrasion will remain after the initial surface roughness has been polished, potentially leading to increased wear over time [30]. For soft or layered nanoparticles, there is the possibility that friction may be reduced through exfoliation (Fig. 1c), whereby the nanoparticles break down into a solid lubricating film, which reduces contact between the surfaces and facilitates low friction through sliding between its layers [29].

The prevalence of each of these mechanisms is expected to be highly situation-specific [1] and depend on variables including the nanoparticle's material, size, shape, hardness, coverage as well as the prevailing temperature, pressure, and sliding velocity. For example, in situ high-resolution scanning electron microscope (HRSEM) [29] and high- 
resolution transmission electron microscope (HRTEM) [31] images of inorganic fullerene $\left(\mathrm{MoS}_{2}\right)$ nanoparticles during sliding have suggested that their friction reduction mechanism consists of a mixture of sliding/rolling at low pressure and exfoliation at high pressure. $\mathrm{CNO}$ has a similar layered structure to inorganic fullerenes, and a thus a similar friction reduction mechanism might be expected. However, HRTEM images during sliding showed that $\mathrm{CNO}$ nanoparticles mostly remain spherical after being subjected to boundary friction experiments $[19,23]$. This suggests that CNO nanoparticles probably achieve low friction by maintaining separation of the contact surfaces and sliding/rolling between them rather than through exfoliation to a graphitic film. The strong diamond structure of CND makes exfoliation even less likely; therefore, they are likely to have a similar mechanism to CNO. Indeed, HRSEM analysis has indicated that CNDs remain intact and become embedded into steel surfaces, increasing their toughness and reducing adhesion from asperity contact $[9,17]$. One might anticipate that indentation of the nanoparticles into the surface might result in increased wear [30]; however, CND and CNO have consistently shown reduced wear in boundary friction experiments $[9,17,19,23]$, indicating that the benefit of asperity separation is more significant than any nanoscale wear caused by nanoparticle indentation. It has also been suggested that the friction reduction of CND may be mainly due to accelerated running-in through polishing of surface asperities [30], though this has not been experimentally corroborated. The uncertainty in the friction reduction mechanism of CND and CNO has certainly contributed to slow commercial uptake of these additives [1,30], making it critical to better understand their nanoscale behaviour before they can be more widely implemented.

Nonequilibrium molecular dynamics (NEMD) simulations have been used to supplement experimental analysis and provide insight into the nanoscale friction reduction mechanisms of both CND and CNO. NEMD simulations of multiple CNOs between diamond-like-carbon (DLC) surfaces have shown that $\mathrm{CNO}$ are able to maintain asperity separation at high pressure $(5.0 \mathrm{GPa})$ and facilitate low friction through a sliding/rolling mechanism [23, 24]. Similarly, NEMD simulations of single CND nanoparticles on $\alpha$-iron surfaces at $1.0 \mathrm{GPa}$ suggested that they are able to effectively reduce friction through the same mechanism [32]. During these simulations, CNO did not indent into the hard DLC surface [23, 24]; however, CND significantly indented the softer $\alpha$-iron surface, leading to a much higher friction coefficient [32]. This suggests that the relative hardness of the nanoparticle and the surface may have a significant influence on the friction and wear behaviour. Prior to the current study, no direct comparison between
CNO and CND has been conducted using NEMD simulations.

The aim of this study is to use atomistic NEMD simulations to shed further light on the friction reduction mechanisms of carbon nanoparticle additives on $\alpha$-iron surfaces, chosen as a model for steel which is generally used in experiments and applications. Two types of nanoparticle will be simulated: (1) a $\mathrm{CNO}$, modelled by four layers of nested fullerenes $\left(\mathrm{C}_{60} @ \mathrm{C}_{240} @ \mathrm{C}_{540} @ \mathrm{C}_{960}\right)$, with a diameter of approximately $3 \mathrm{~nm}$, and (2) a CND, modelled by a diamond sphere, also with a diameter of approximately $3 \mathrm{~nm}$. These particle diameters, although rather small due to computational constraints, still lie well within the experimentally relevant range [9]. Simulating both CND and CNO allows us to assess their relative effectiveness under identical conditions, as well as to uncover any differences in their friction reduction mechanisms.

The NEMD simulations will be conducted under a wide range of conditions, with variables including surface coverage, applied normal pressure, and sliding velocity. This should reveal a more complete picture of how carbon nanoparticle additives operate at the nanoscale under boundary lubrication conditions. The fact that NEMD simulations are limited to the nanoscale means that it is not feasible for them to recreate all situations where the nanoparticles may provide a reduction in friction and wear (e.g. Fig. 1d); however, they can yield unique insights into the nanoscale tribological behaviour of the nanoparticles confined between asperities, which are inaccessible to experiments [33]. The remainder of this manuscript will be presented as follows; first, details of the simulation methodology are given, this is followed by discussion of the results in the context of the application of $\mathrm{CNO}$ and CND as lubricant additives, before final conclusions are drawn.

\section{Methodology}

Classical NEMD simulations were performed in LAMMPS [34]. In all of the simulations, two atomically smooth (100) slabs of $\alpha$-iron with dimensions ( $x y z$ ) of approximately $9.0 \times 9.0 \times 3.0 \mathrm{~nm}$ were used as the substrates, representing a single asperity contact (Fig. 2a). Nanoparticles were confined between the two slabs, and periodic boundary conditions were applied in the $x$ - and $y$-directions (Fig. 2c). No base oil molecules were included in these simulations since, under high-pressure boundary conditions, we can assume that most of them will be squeezed out from between asperities [27, 28], and thus the nanoparticles will support the majority of the load. Adding 


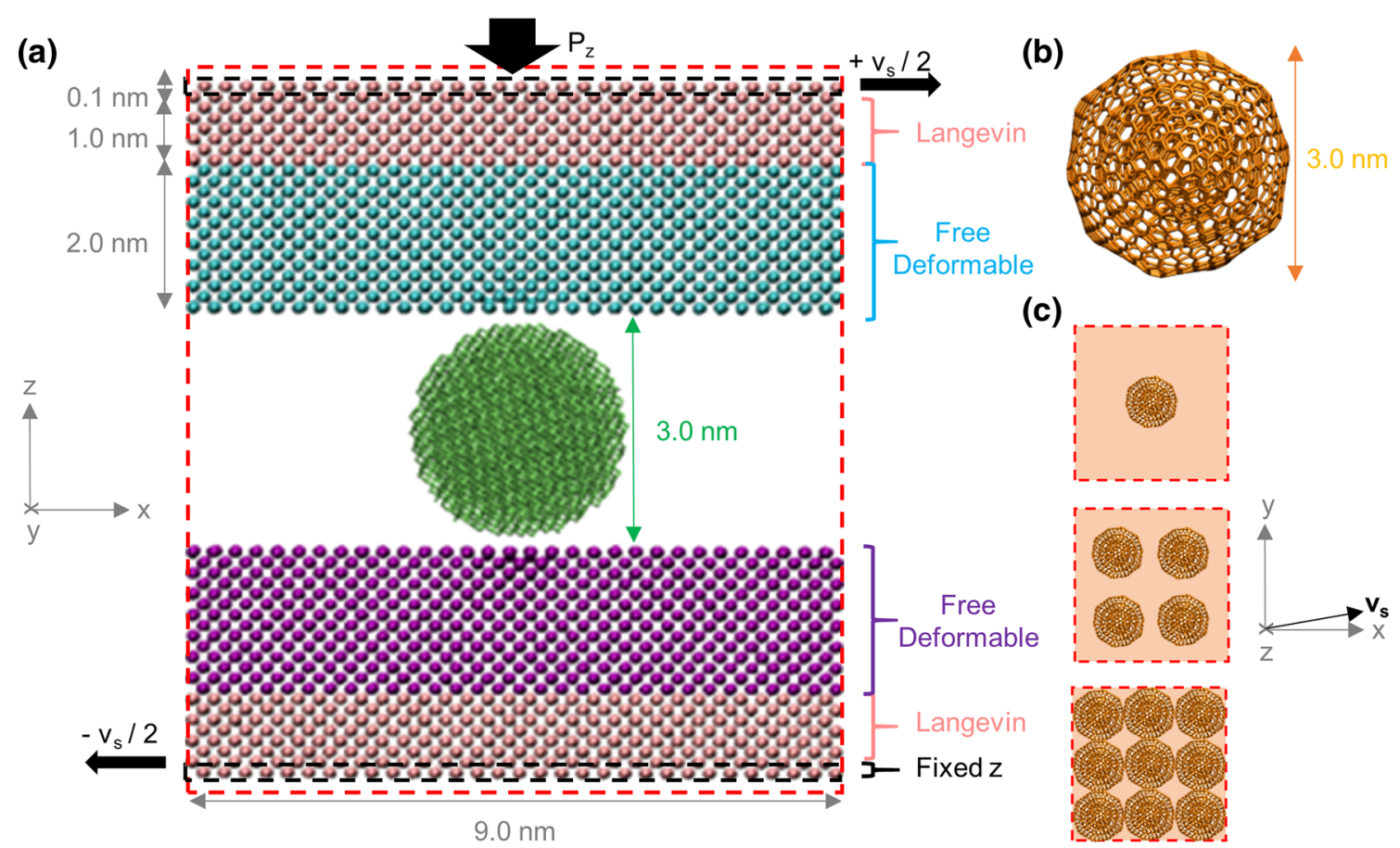

Fig. 2 a Image of representative system set-up for NEMD simulations $(\mathrm{CND}, \theta=0.11)$. Carbon-carbon bonds are shown in green for $\mathrm{CND}$ and orange for $\mathrm{CNO}, \mathrm{Fe}$ atoms are shown in pink for the thermostatted and fixed atoms and cyan (top) or purple (bottom) for the deformable atoms. The red dotted lines indicate periodic

an arbitrary number of base oil molecules in these simulations would be counterproductive, since the periodic boundary conditions mean that molecules cannot be squeezed out from between asperities as they would be in experimental systems and they would therefore provide additional, unphysical load support.

The $\mathrm{C}-\mathrm{C}$ interactions in $\mathrm{CNO}$ and $\mathrm{CND}$ are modelled using an updated version of the adaptive intermolecular reactive empirical bond order (AIREBO) potential [35], known as AIREBO-M [36]. AIREBO-M uses the same functional form as the hydrocarbon second-generation REBO2 potential [37] for covalent $\mathrm{C}-\mathrm{C}$ interactions, with an additional Morse term to represent the Van der Waals interactions. The Morse potential is 'switched off' at the onset of $\mathrm{C}-\mathrm{C}$ bond formation in order to prevent disruption of the REBO2 energies. The Morse potential replaces the Lennard-Jones potential used in the original AIRBEO, which has been shown to yield unphysical results for carbon systems subjected to high pressures [36]. The use of AIREBO-M rather than AIREBO is expected to more accurately reproduce the interlayer distances in the $\mathrm{CNO}$ nanoparticles, as well as interactions between neighbouring carbon nanoparticles, under the high normal pressures applied.

In order to accurately model plastic deformation within the slab, the $\mathrm{Fe}-\mathrm{Fe}$ interactions are modelled using the boundaries. Rendered using VMD [44]. b Image of four-layer CNO particle(C60@C240@C540@C960). c Schematic showing the nanoparticle coverages simulated; $\theta=0.11,0.44$, and 1.00. Velocity, $v_{\mathrm{s}}$, applied at $10^{\circ}$ to the $x$-axis of the periodic box (Color figure online)

embedded atom model (EAM) potential [38]. Iron and steel surfaces quickly become oxidised when exposed to air, which significantly reduces the adhesion force between contact surfaces in experimental systems [39]. Therefore, a Lennard-Jones potential was used for $\mathrm{Fe}-\mathrm{Fe}$ interactions between atoms in opposing slabs (Fig. 2a) in order to mimic the reduced adhesion between oxidised surfaces [40]. The Lennard-Jones parameters, $\varepsilon=0.02045 \mathrm{eV}$ and $\sigma=3.21 \AA$, were used for this interaction, as have been successfully utilised in previous tribological NEMD simulations [39, 40].

The $\mathrm{Fe}-\mathrm{C}$ Van der Waals interactions are also modelled using a Lennard-Jones potential. The Lennard-Jones parameters used here, $\varepsilon=0.02495 \mathrm{eV}$ and $\sigma=3.70$, were parameterised for MD simulations of carbon nanotube growth on iron surfaces [41] and have since been successfully applied in tribological NEMD simulations of CNDs between iron surfaces [32] as well as nanoscratching of iron by diamond tips $[42,43]$.

All systems were constructed using the materials and processes simulations platform (MAPS) from Scienomics SARL. A representative example of the systems simulated in this study is shown in Fig. 2a.

The MD equations of motion were integrated using the velocity Verlet algorithm with an integration time step of 1.0 fs. A Langevin thermostat [45], with a time relaxation 
constant of $0.1 \mathrm{ps,}$ was used in all of the simulations in order to maintain a temperature of $300 \mathrm{~K}$. The thermostat acted only on the atoms in the outer $10 \AA(z)$ of each slab (Fig. 2a) and was applied in the direction perpendicular to the both the sliding and compression $(y)$ [28, 32]. This method is known to be advantageous over direct thermostatting of systems in confinement, which has been shown to significantly affect their behaviour during sliding [46].

A wide phase space was mapped in order to capture any changes in the friction reduction mechanism of the nanoparticles under boundary conditions. Specifically, the fractional coverage of the nanoparticles, $\theta$, the applied normal pressure, $P_{z}$, and sliding velocity, $v_{\mathrm{s}}$, were all varied independently. A maximum coverage $(\theta=1.00)$ was simulated by including nine nanoparticles in direct contact with each other in a primitive cubic arrangement between the slabs. A medium $(\theta=0.44)$ and low $(\theta=0.11)$ coverage were also simulated by including four and one nanoparticles between the slabs, respectively. The nanoparticles were initially positioned to give maximum possible separation from one another (Fig. 2c). A simulation with no nanoparticles between the sliding surfaces was also conducted in order to estimate the percentage friction reduction achieved with CNO and CND.

The nanoparticles were initially positioned $3 \AA$ from the slabs prior to energy minimisation. The systems were energy minimised, compressed, and equilibrated before sliding was applied. After energy minimisation, the outer shell of CNO became slightly faceted (Fig. 2b) [11], whereas CND remained entirely spherical (Fig. 2a). The normal pressure, $P_{z}=1.0,2.0,3.0,4.0$, and $5.0 \mathrm{GPa}$, was applied to the slab by adding a constant force in the $z$-direction to the outermost layer of atoms in the top slab whilst keeping the outermost layer of atoms in the bottom slab fixed in the $z$-direction, as is common in confined NEMD simulations [28, 32]. The target pressure was reached gradually by increasing the normal force from that corresponding to $P_{z} / 100, P_{z} / 20, P_{z} / 10, P_{z} / 2$ and finally $P_{z}$ during $100 \mathrm{ps}$ increments. As the normal pressure was increased, the slab separation initially varied in a damped harmonic manner, so sliding was not applied until a constant average slab separation was obtained at the target pressure, $P_{z}$.

A range of sliding velocities, $v_{\mathrm{s}}=5,10$, and $20 \mathrm{~m} \mathrm{~s}^{-1}$, were applied in order to monitor any change in the friction coefficient as well as the rolling/sliding motion of the nanoparticles. Whilst lower sliding velocities are desirable to match those used in boundary friction experiments (typically $\mathrm{mm} \mathrm{s}^{-1}$ ), they are not yet accessible using atomistic NEMD simulations of this scale [28]. When compressive oscillation became negligible, a velocity of $v= \pm v_{\mathrm{s}} / 2$ was added to the outermost layer of atoms in each slab (Fig. 2). In the low coverage and high-pressure simulations, the nanoparticles ploughed through the surface to produce nanoscale wear tracks. Therefore, when the sliding velocity was applied parallel to the $x$-axis of the periodic box yield, the nanoparticles reworked their own wear tracks as they passed through periodic boundaries, leading to an unphysical decrease in the friction coefficient. Consequentially, for all of the results given below, the sliding velocity was applied at a $10^{\circ}$ angle from the $x$-axis of the periodic box (Fig. 2c), which was sufficient to prevent the nanoparticles from reworking their own wear tracks after passing through the periodic boundary [33]. Sliding simulations were conducted for $10-40 \mathrm{~ns}$, depending on the sliding velocity. All simulations were run for long enough to yield a sufficient sliding distance $(20 \mathrm{~nm})$ in order to obtain a converged friction coefficient, with an uncertainty less than $10 \%$. This uncertainty was estimated as the standard deviation between the block average friction coefficient values, which were calculated every $0.5 \mathrm{~nm}$ after $2.0 \mathrm{~nm}$ of sliding. It was also confirmed for a representative sample that the standard deviation between ensemble average friction coefficients from three independent trajectories yielded a similar level of uncertainty.

\section{Results and Discussion}

In this study, we use NEMD to provide unique insights into the nanoscale friction and wear reduction mechanism of carbon nanoparticles CND and CNO. The kinetic friction coefficient, $\mu$, was obtained using the extended AmontonsCoulomb law under the high load approximation: $\mu=F_{\mathrm{L}} /$ $F_{\mathrm{N}}$, where $F_{\mathrm{L}}$ and $F_{\mathrm{N}}$ are, respectively, the average total lateral and normal forces acting on the outer layer of atoms in each slab. Variations in the friction coefficient with sliding distance are presented for CND and CNO under a range of coverages, pressures, and sliding velocities. The variation in the average friction coefficient with pressure and indentation depth are also presented and linked to macroscopic predictions. The indentation depth of the nanoparticles into the slabs, temperature profiles of the systems during sliding, and angular velocities which indicate whether the nanoparticles are rolling or sliding are also included in order to gain more insight into the friction reduction mechanisms of the nanoparticles. All results are presented as block averages, calculated every $0.5 \mathrm{~nm}$ of sliding. Simulation snapshots are included to enable visualisation of important changes in behaviour under different simulation conditions. The results section is divided into three sections corresponding to the variation in friction and wear due to the three main variables investigated in this study: the nanoparticle coverage (3.1), the normal pressure (3.2), and the sliding velocity (3.3). 


\subsection{Effect of Nanoparticle Coverage}

Results for NEMD simulations with no nanoparticles between the slabs are presented first in order to enable estimation of the benefits of different coverages of $\mathrm{CNO}$ and CND.

Figure 3 shows that when $\alpha$-iron slabs are slid against one another without nanoparticles between them, the friction behaviour varies significantly with sliding distance. Initially, there is a sharp increase to a high friction coefficient $(\mu>5)$ as a result of the very high commensurability of the opposing surfaces which 'locks' them together (Fig. 3). After around $1 \mathrm{~nm}$ of sliding, a transfer film forms between the opposing surfaces (Fig. 3) [32]. Here, the friction coefficient decreases [39, 47] and converges to $\mu \approx 1$, which is comparable to the experimentally observed value for the dry sliding of steel surfaces [22, 48], thus validating this surface model for the reproduction of experimental friction coefficients.

Figure 4 shows the change in the friction behaviour when $\mathrm{CNO}$ and $\mathrm{CND}$ nanoparticles are present at various coverages when $P_{z}=1.0 \mathrm{GPa}$. Comparing Figs. 3, 4 indicates that the friction coefficient is reduced considerably in the presence of CNO and CND at all nanoparticle coverages considered. Figure 4 also shows that the friction coefficient varies significantly with nanoparticle coverage. Since the apparent contact area decreases at lower coverages, the local pressure on each particle increases. The nanoparticles do not indent into the slabs at 0.44 coverage and 1.00 coverage (Fig. 5), which leads to very low friction coefficients $(<0.05)$ that remain relatively constant through the course of the sliding simulation (Fig. 4). Here, the friction coefficient arises purely from resistance to rolling/ sliding due to Van der Waals interactions between the nanoparticle and the slabs, which dislodges surface atoms

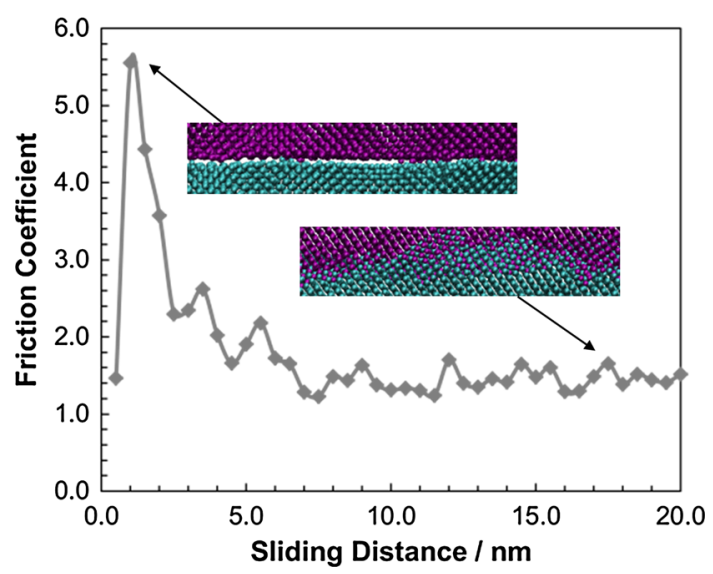

Fig. 3 Dry sliding of $\alpha$-iron slabs, variation in the friction coefficient with sliding distance. For $P_{z}=1.0 \mathrm{GPa}$ and $v_{\mathrm{s}}=10 \mathrm{~m} \mathrm{~s}^{-1}$. Inset images show interface after 200 and 2000 ps ( 2 and $20 \mathrm{~nm}$ of sliding) from their lattice positions, thus increasing the shear stress. At 0.11 coverage, the friction coefficient sharply increases to around 0.35 for $\mathrm{CNO}$ and 0.40 for CND and then converges after approximately $2 \mathrm{~nm}$ of sliding. This is because, at 0.11 coverage, there is significant indentation of the nanoparticles into the surface, which plough nanoscale wear tracks during sliding (Fig. 5). The ploughing action of the nanoparticles causes plastic deformation within the slab, leading to an additional, much larger, contribution to the friction coefficient [49]. As has been observed experimentally [19, 23], CNO yields a lower friction coefficient than CND in these simulations. Experiments which have shown CND to be more effective in reducing friction [17] probably achieved better dispersion of CND than CNO, leading to a higher surface coverage.

The 0.11 coverage $\mathrm{CNO}$ and CND systems show a slight increase in the friction coefficient after one pass of the surface $(\approx 9 \mathrm{~nm})$, due to contact of the nanoparticles with wear debris ejected during the first pass of the surface. However, after $\approx 2 \mathrm{~nm}$ more sliding, the friction coefficient returns to the same as value before passing through the periodic boundary, suggesting that any atomic-scale roughness present on the surface will be removed by the nanoparticles and will have a negligible influence on the friction coefficient. This also indicates that surface polishing due to the presence of CNO and CND may be another contributor to low friction, as has been suggested previously from experimental results $[12,16]$.

The friction-coverage behaviour agrees well with boundary friction experiments which, at low concentrations, show a decrease in friction coefficient when the concentration of CND or CNO is increased [21, 25]. At 0.11 coverage (Fig. 4 ), the friction coefficient is reduced by approximately $75 \%$ relative to when no nanoparticle is present (Fig. 3). This percentage friction reduction agrees broadly well with results of boundary friction experiments which used carbon nanopearls (larger, $\approx 150 \mathrm{~nm}$ layered nanoparticles) as a solid lubricant ( $\approx 85 \%$ ) [22], as well as when $\mathrm{CNO}$ and CND are used as additives in a base oil $(\approx 50 \%)[12,23]$. The very low friction coefficients and zero wear observed at 0.44 and 1.00 coverage in these simulations have not been detected in boundary friction experiments of $\mathrm{CNO}$ and CND. Whilst this can partly be attributed to the lack of viscous friction from the presence of a base oil in these simulations, it also suggests that high nanoparticle coverages were probably not reached in the experiments. This is because, although nanoparticle coverage initially increases with concentration, at higher concentrations the nanoparticles begin to agglomerate and become too large to enter the contact, leading to a reduction in surface coverage [15]. Therefore, CNO and CND nanoparticles may be even more effective in reducing boundary friction and wear should better dispersion 
Fig. 4 Effect of nanoparticle coverage, $\theta$, on the friction coefficient. For $P_{z}=1.0 \mathrm{GPa}$ and $v_{\mathrm{s}}=10 \mathrm{~m} \mathrm{~s}^{-1}$
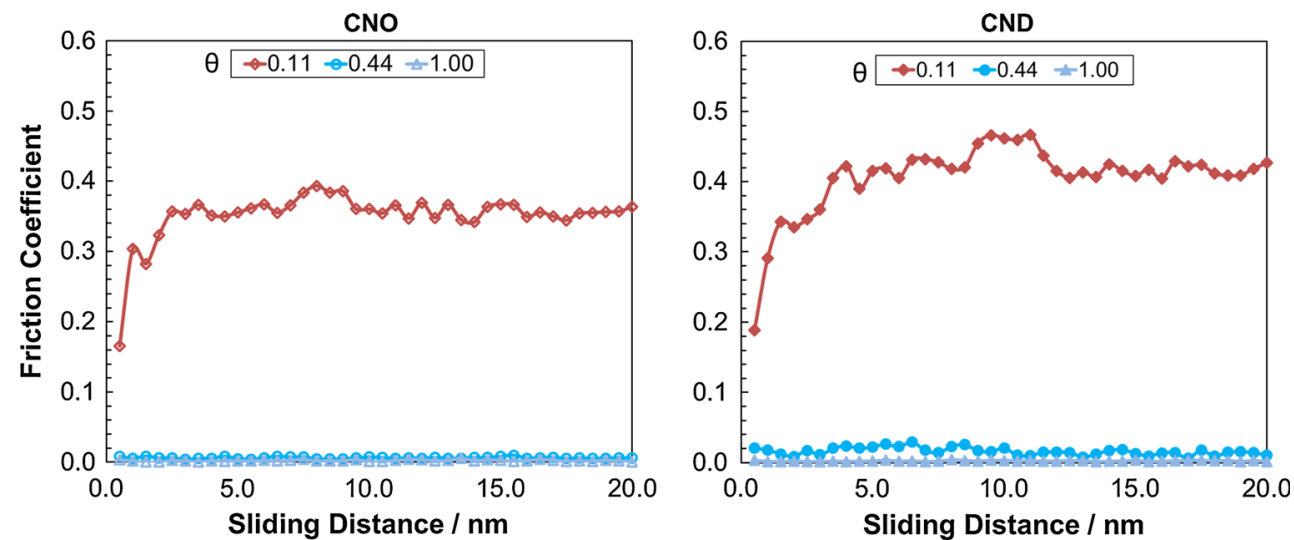

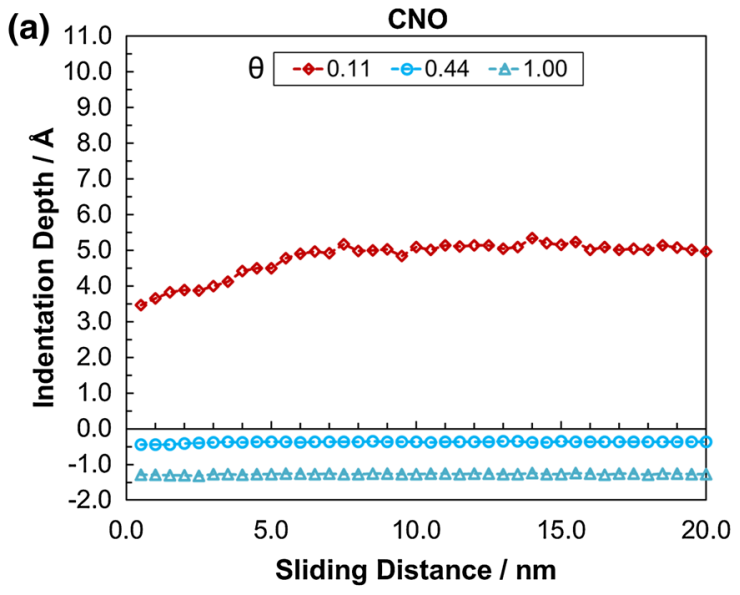

(b)

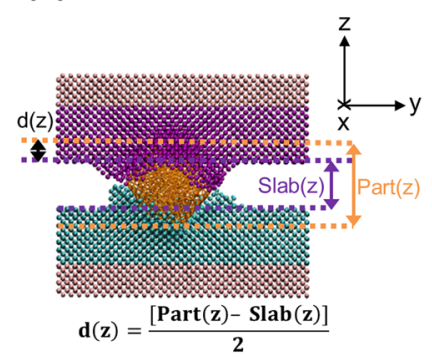

(c)

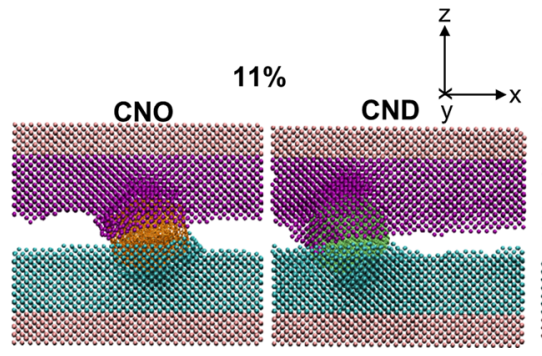

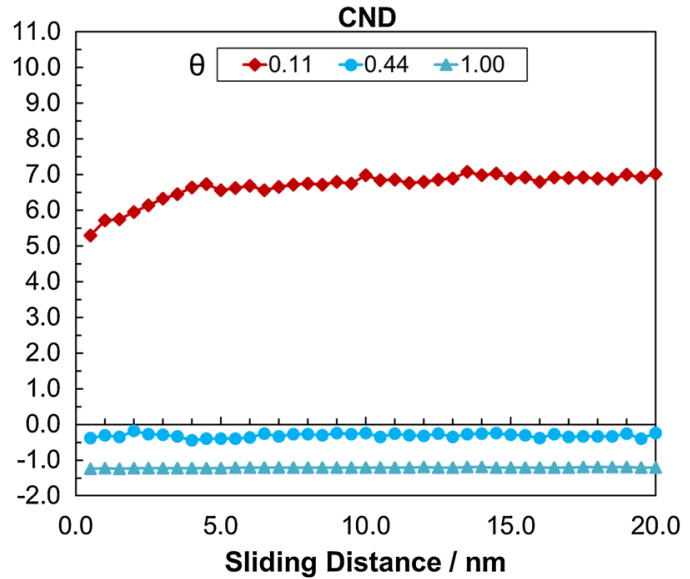

Sliding Distance / nm
Fig. 5 a Effect of nanoparticle coverage, $\theta$, on the indentation depth, $\mathrm{d}(z)$, of nanoparticles into slabs. For $P_{z}=1.0 \mathrm{GPa}$ and $v_{\mathrm{s}}=10 \mathrm{~m} \mathrm{~s}^{-1}$. b Schematic viewed along $x$ showing the ploughing of the wear track by

methods to be developed in order to yield high surface coverages.

Figure 5a shows the indentation depth, $\mathrm{d}(z)$, of the nanoparticles into each of the $\alpha$-iron slabs. This is monitored throughout the sliding simulation by subtracting the $z$-extension of the nanoparticle, $\operatorname{Part}(z)$, from the $z$-separation of the outer layer of undisturbed atoms in the top and bottom slabs, $\operatorname{Slab}(z)$, and dividing this value by two, as shown in Fig. 5b. Slab $(z)$ excludes slab atoms which form part of the wear debris during the ploughing of the nanoparticles (Fig. 5b) [33]. Part(z) remains relatively
$\mathrm{CNO}$ after $10 \mathrm{~nm}$ of sliding; indicating definitions of $\operatorname{Slab}(z), \operatorname{Part}(z)$, and $\mathrm{d}(z)$. c Images of 0.11 and 1.00 coverage systems along $y$ after $10 \mathrm{~nm}$ of sliding

constant through the course of the simulation for both nanoparticles, but is fractionally lower for CNO (28 $⿱$ ) than CND (30 $\AA$ ), suggesting that the CNO particles compress slightly under the applied normal pressure $(1.0 \mathrm{GPa})$.

When $\theta=0.44$ and $\theta=1.00$, the indentation depth value in Fig. 5a is negative, indicating that there is no overlap of the average $z$-positions of the outermost layer of atoms in the nanoparticles and the slabs. This results in zero wear and a very low friction coefficient (Fig. 4). The average position of the nanoparticles is slightly closer to 
the slabs when $\theta=0.44$ than when $\theta=1.00$, as well as for CND relative to $\mathrm{CNO}$, leading to stronger slab-nanoparticle Van der Waals interactions and somewhat higher friction coefficients (Fig. 4). At 0.11 coverage, there is significant indentation of the $\alpha$-iron slabs by the nanoparticles, which plough atomic-scale wear tracks during sliding (Fig. 5). The nanoparticles are indented into the slabs even before the onset of sliding, CND ( $5 \AA$ ) to a slightly greater extent than CNO ( $3 \AA$ ). As the nanoparticles plough through the slabs, indentation increases slightly, with CND (7 remaining more indented than CNO (5 ̊). This suggests that the lower friction coefficient for CNO relative to CND at 0.11 coverage (Fig. 4) is a result of reduced indentation into the slab (Fig. 5), due to its softer layered structure.

An important observation is that for both CNO and CND nanoparticles when $\theta=0.11$, plastic deformation of the surface around the nanoparticle occurs rather than plastic deformation of the nanoparticle itself. There was no evidence of exfoliation of CNO in any of the simulations, which is consistent with HRTEM images taken after boundary friction experiments [23] and high-pressure Raman spectroscopy [50, 51] which suggest very high compressive strength owing to the concentric nested structure of CNO.

In boundary lubrication, contact between opposing surfaces leads to adhesion and high friction, which can in turn lead to an increase in surface temperature. Temperature profiles in $z$ are shown for when no nanoparticle is present (Fig. 6a) and with $\mathrm{CNO}$ and $\mathrm{CND}$ at 0.11 coverage (Fig. 6b), at the start (0-200 ps) and end (1800-2000 ps) of the sliding simulations. The temperature profiles show the expected parabolic shape for boundary-thermostatted systems during sliding, with a maximum temperature in the centre of the system [52]. Both nanoparticle systems exhibit lower maximum temperatures (CND $315 \mathrm{~K}$, CND $305 \mathrm{~K})$ compared to when no nanoparticle is present $(350 \mathrm{~K})$ at the end of the sliding simulation. The CND system showed a large initial increase in temperature within the nanoparticle due to the high thermal conductivity of diamond; however, the maximum temperature at the end of the sliding simulation is similar to that for $\mathrm{CNO}$, and much lower than when no nanoparticle is present. It should be noted that the exclusion of electronic effects in classical MD simulations leads to an underestimation of metal thermal conductivity, and is responsible for the relatively high-temperature gradients within these systems [33]. Nonetheless, a similar reduction in surface temperature as found in these simulations has also been observed in previous experimental studies. For example, Ivanov et al. [14] observed a reduction in surface temperature from approximately 400 to $340 \mathrm{~K}$ for formulations which included CND compared to a pure base oil. From these simulations, this reduction in surface temperature can be attributed to the reduction in contact of opposing surfaces, which leads to a decrease in frictional heating, as exemplified in by the lower friction coefficients in the nanoparticle-containing systems (Fig. 3 vs. 4). The slight reduction in the maximum temperatures of systems containing CNO relative to CND at the end of the sliding simulation can be rationalised using the same arguments (Fig. 4).

\subsection{Effect of Normal Pressure}

In order to further probe the friction and wear behaviour of $\mathrm{CNO}$ and CND from the onset of indentation, variable pressure simulations were also conducted. Figure 7 shows the variation in the friction coefficient with sliding distance when $\theta=0.44$ and for pressures between 1.0 and 5.0 GPa. The slight increase in the friction coefficient after $9 \mathrm{~nm}$ is not identifiable in the 0.44 coverage results (Fig. 7) because there are multiple particles which pass through periodic boundaries after different sliding distances. From Fig. 7, it is clear that CND yields a higher friction coefficient than $\mathrm{CNO}$ at all pressures simulated. Moreover, the friction behaviour suggests that the onset of indentation at
Fig. 6 Time-averaged temperature profile in $z$ for the system after 200 and 2000 ps (2 and $20 \mathrm{~nm}$ of sliding) for when no nanoparticle is present (a) and for $\theta=0.11 \mathrm{CNO}$ and CND (b), for $P_{z}=1.0 \mathrm{GPa}$ and $v_{\mathrm{s}}=10 \mathrm{~m} \mathrm{~s}^{-1}$ (a)

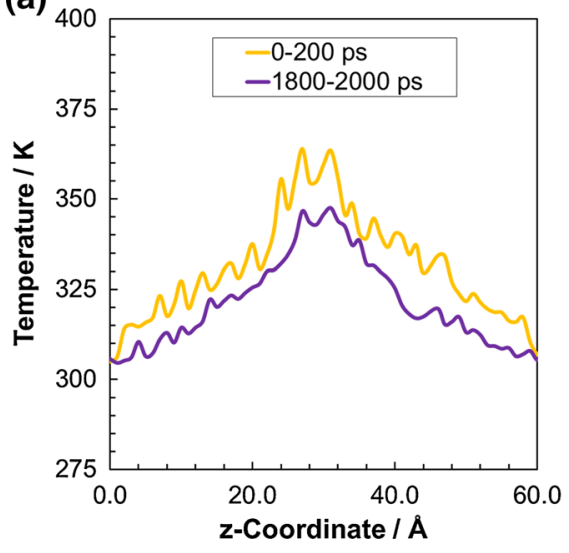

(b)

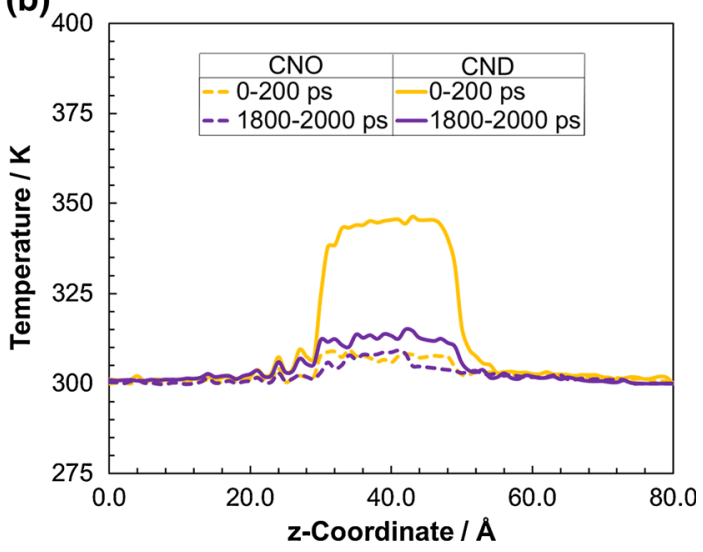


Fig. 7 Effect of pressure, $P_{z}$, on the friction coefficient. For $P_{z}=1.0-5.0 \mathrm{GPa}, \theta=0.44$ and $v_{\mathrm{s}}=10 \mathrm{~m} \mathrm{~s}^{-1}$
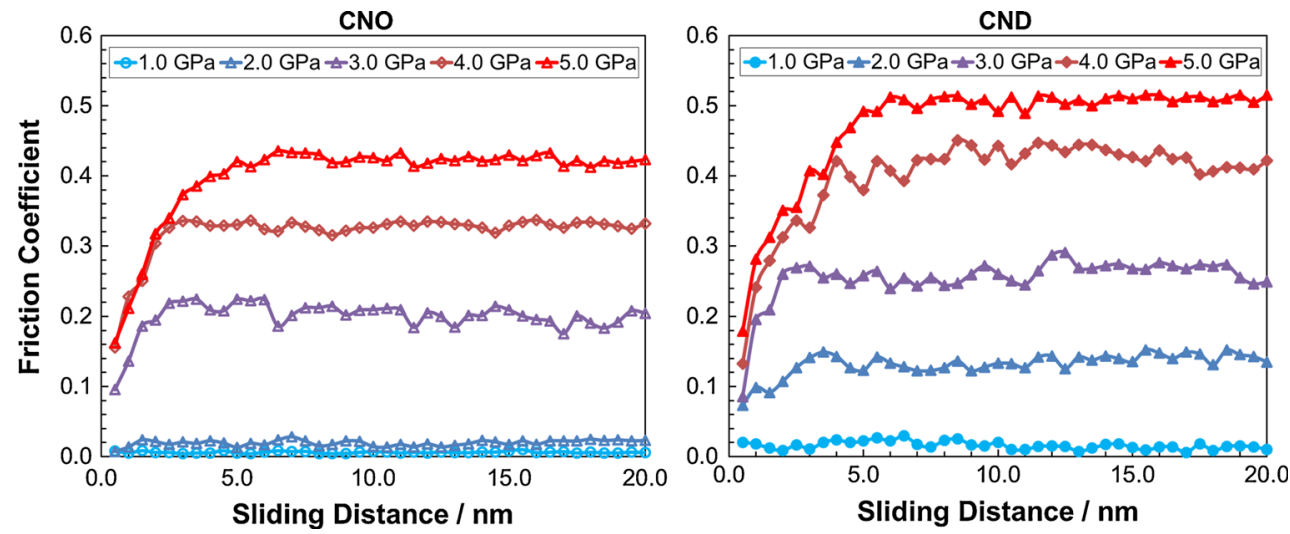

0.44 coverage occurs at lower pressure for CND (2.0 GPa) than for CNO (3.0 GPa).

Figure 8 clearly indicates the differences in nanoparticle indentation depth between the CNO and CND systems. For example, at $2.0 \mathrm{GPa}$, the CND nanoparticles become indented into the slabs whilst the softer CNO nanoparticles do not. At 3.0 GPa and above, both CND and CNO nanoparticles become indented into the slab; however, CND nanoparticles to a greater extent than CNO. Even at the highest pressure $(5.0 \mathrm{GPa})$, the undisturbed layers of atoms in the opposing slabs remain well separated $(\operatorname{Slab}(z) \approx 10 \AA)$, though some wear debris atoms ejected from opposing slabs do come into contact. This contact seems to have only a minimal effect on the friction coefficient (Fig. 7), and ploughing of the nanoparticles remains the dominant factor. The contribution to friction from adhesion between atoms in opposing slabs may become more significant at higher pressures, or lower coverages than those simulated here, when the nanoparticles are no longer able to maintain separation of opposing slabs.

The 0.44 coverage at $4.0 \mathrm{GPa}$ (Fig. 7) and the 0.11 coverage at 1.0 GPa (Fig. 5) are equivalent in terms of the force on each nanoparticle. The differences in the friction and wear behaviour between these two cases show that the effect of pressure and coverage is subtly different. At 0.44 coverage, there is slightly less indentation into the surface
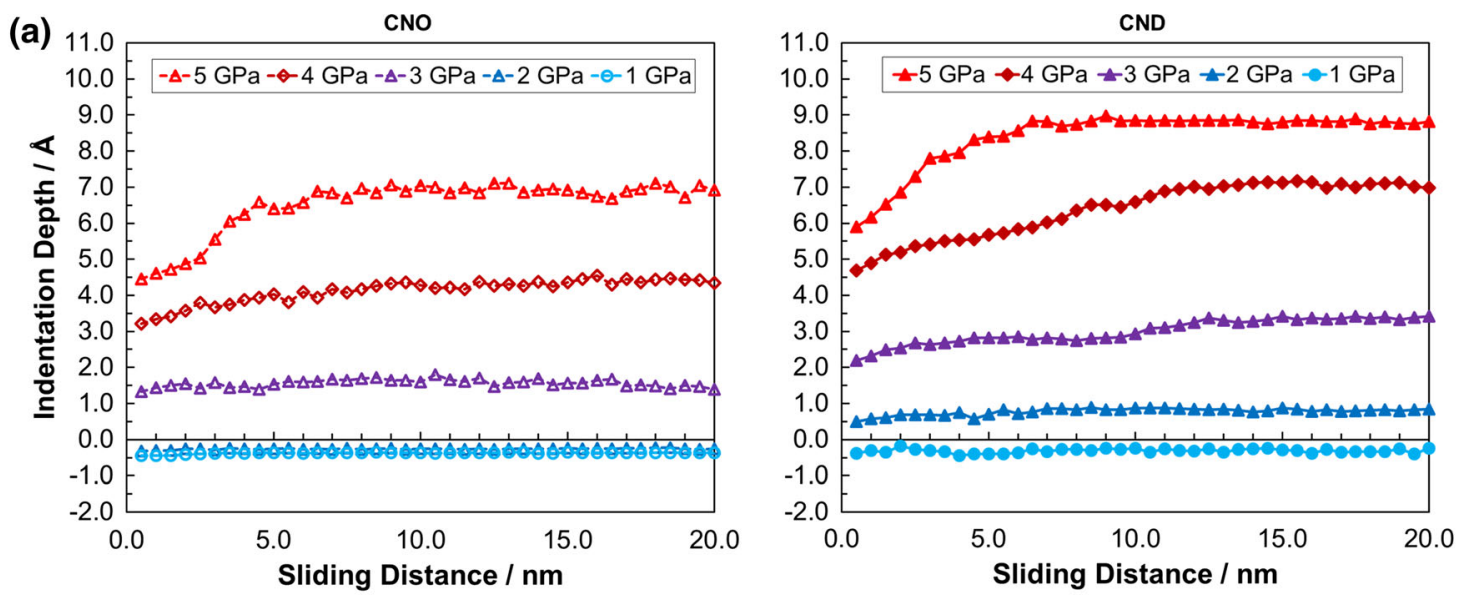

(b)
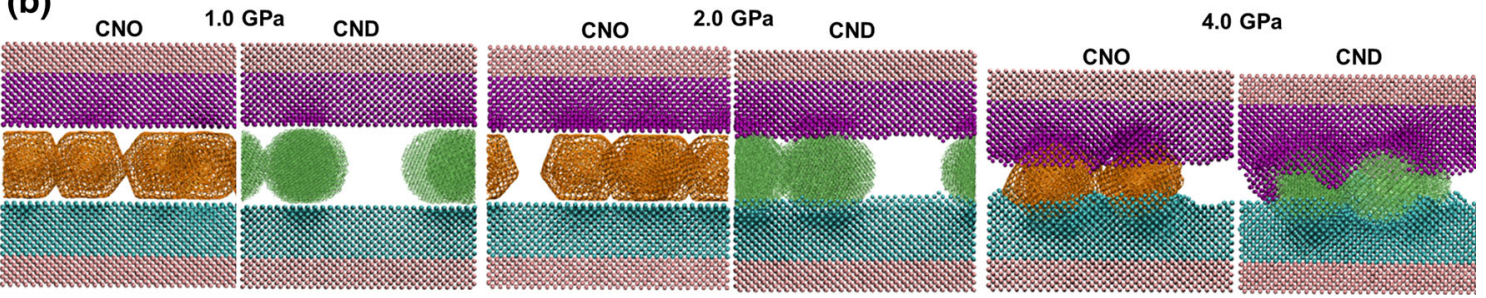

Fig. 8 a Effect of pressure, $P_{z}$, on the indentation depth, $\mathrm{d}(z)$. For $P_{z}=1.0-5.0 \mathrm{GPa}, \theta=0.44, v_{\mathrm{s}}=10 \mathrm{~m} \mathrm{~s}^{-1}$. b Images of 0.44 coverage systems along $y$ after $10 \mathrm{~nm}$ of sliding at different pressures 
than at $\theta=0.11$ due to a more even distribution of the force over the slabs. This leads to a very slightly $(5 \%)$ lower friction coefficient at 0.44 than at 0.11 coverage at equivalent pressure per nanoparticle.

Figure 9a shows the variation in the average friction coefficient with pressure. Above 2.0 GPa for $\mathrm{CNO}$ and 1.0 GPa for CND, the friction coefficient increases rapidly with pressure due to increasing indentation of the nanoparticles. This increase in friction coefficient with pressure has been also observed in previous NEMD simulations of hard nanoparticles [33] as well as in nanoscratching simulations of $\alpha$-iron surfaces by diamond tips $[42,43]$. This relationship has also been observed experimentally for CNO additives [21, 26]; however, other boundary friction experiments have shown increasing wear, but decreasing friction, with increasing pressure [23]. In these experiments, the decrease in friction with increasing pressure was evident both in the presence and in the absence of nanoparticles in a base oil [23]. This is probably because, at higher pressure, there is increased contact of asperities, which become smoothed over experimental timescales, resulting in increased wear but decreased friction. This experimentally observed decrease in friction coefficient with increasing pressure may sometimes supersede the increase which has been observed in these and previous [33] NEMD simulations of hard nanoparticles.

From Fig. 9b, it is clear that, after the onset of indentation, the nanoparticle indentation depth increases almost linearly with pressure, as is anticipated from previous nanoindentation experiments [53] and simulations [42, 43]. In these current simulations, CND indents more than $\mathrm{CNO}$ at equal pressure, which may be due to higher hardness. The normal hardness of a material, $H_{\mathrm{N}}$, can be directly related to its indentation depth through: $H_{\mathrm{N}}=F_{\mathrm{N}} / A_{\mathrm{N}}$, where $A_{\mathrm{N}}$ can be estimated for a spherical indenter through
$A_{\mathrm{N}}=\pi\left[R^{*}-\left(R^{*}-\mathrm{d}(z)\right)^{2}\right] . \quad$ Here, $R^{*}$ is the apparent indenter radius, given by: $R^{*}=R+r_{\mathrm{c}}$, where $\mathrm{R}$ is the radius of the nanoparticle ( $15 \AA), r_{\mathrm{c}}$ is the cut-off for the repulsive part of the $\mathrm{C}-\mathrm{Fe}$ potential $(3.8 \AA)$ [43], and $\mathrm{d}(z)$ is the indentation depth. Note that the cut-off for the Van der Waals C-Fe potential is used here purely to assign the radius of the indenter, and no attractive or repulsive forces are considered. Conventionally, this relationship is used to calculate the hardness of the substrate rather than the indenter, but given that the hardness of the $\alpha$-iron slab is the same in both cases, the difference between the contact areas estimated from the indentation depths for CNO and CND at a given pressure can be used to give an approximation of their relative hardness. CND nanoparticles are generally around $25 \%$ more indented than CNO (Fig. 9c), suggesting that CND nanoparticles are approximately $25 \%$ harder than CNO. This $25 \%$ difference in hardness is much less than the difference in hardness between diamond and graphite, suggesting that the relatively high hardness of CNO is mainly due to its concentric nested structure.

The ploughing of wear tracks in these simulations represents a nanoscale wear process, with the amount of wear being proportional to the nanoparticle indentation depth [33]. Purely through the difference in indentation depths in these simulations, $25 \%$ more wear in systems lubricated by CND than systems lubricated by CNO would be expected at equal coverage and pressure. Indeed, this difference is similar to that which has been observed experimentally [23]. It is important to note that both nanoparticles are expected to reduce wear compared to systems not containing nanoparticles, because maintaining separation of opposing slabs and reducing contact of asperities have been shown to be more significant than the nanoscale wear caused by the indentation of the nanoparticles [9, 17, 19, 23]. However, since these processes occur

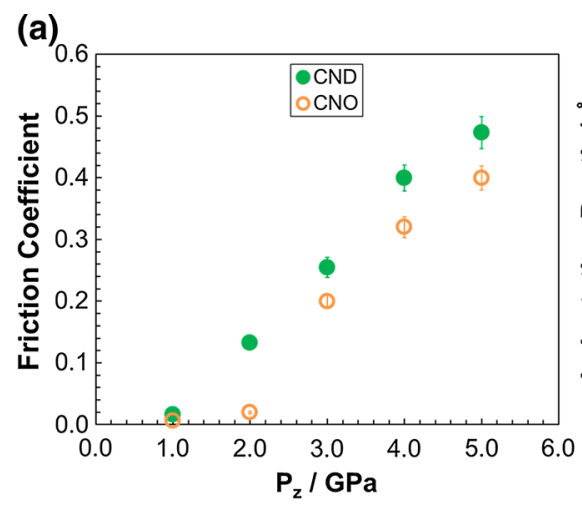

(b)

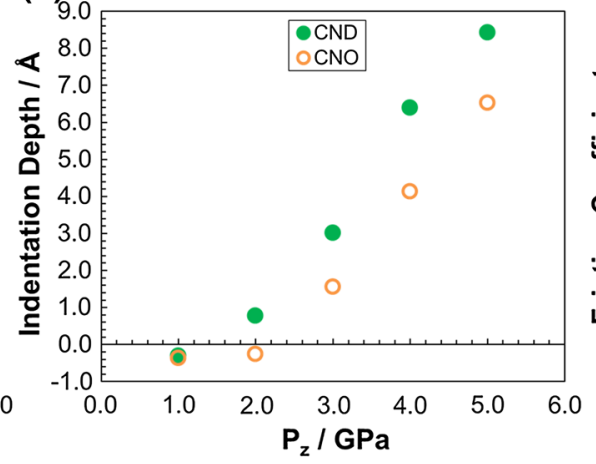

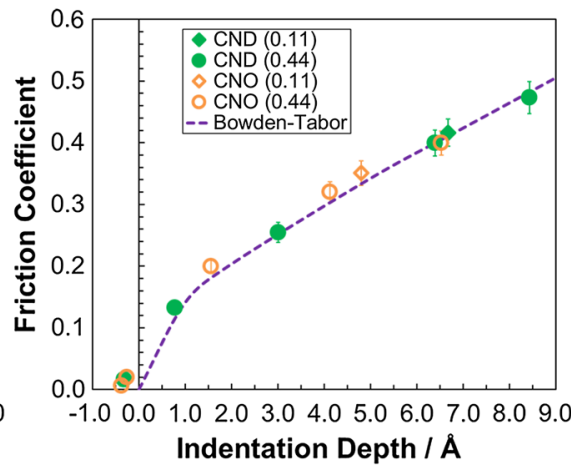

Fig. 9 a Variation in the friction coefficient with normal force. b Variation in the indentation depth with normal force. c Variation in the friction coefficient with the indentation depth. The dotted line in c indicates the Bowden-Tabor prediction from Eq. 1. Error bars indicate the standard deviation between block average friction coefficient values 
on different length scales, it is not possible to quantify this difference using the current simulations.

Figure 9c shows the relationship between the indentation depth and the friction coefficient for all of the 0.44 and 0.11 coverage data. The observed relationship is well predicted by the analytical expression (Eq. 1) developed by Bowden and Tabor [54] to describe the friction coefficient of spherical indenters. The expression assumes that the same material hardness applies in both the normal and lateral directions, which has been confirmed in previous simulations of $\alpha$-iron [43]. This means that the friction coefficient, $\mu$, can be predicted through the ratio of the nanoparticle areas 'seen' by the substrate in the normal and lateral direction, given by:

$\mu=A_{\mathrm{N}} / A_{\mathrm{L}}=\left[2 \theta_{\mathrm{s}}-\sin \left(2 \theta_{\mathrm{s}}\right)\right] /\left[\pi \sin ^{2}\left(\theta_{\mathrm{s}}\right)\right]$

where $\theta_{\mathrm{s}}$ is the semi-angle at the centre of the nanoparticle subtended by the groove and is obtained from $\cos \left(\theta_{\mathrm{s}}\right)=\left(R^{*}-d\right) / R^{*}$. Equation 1 predicts that the friction coefficient will increase with indentation depth since the projected lateral area $\left(A_{\mathrm{L}}\right)$ increases faster than the normal area $\left(A_{\mathrm{N}}\right)$. Using Eq. 1, we calculate the friction coefficient as a function of indentation depth, see the dashed line in Fig. 9c. The simulation results agree well with the calculated friction coefficient values for the range of indentation depths encountered. This suggests that, at the small indentation depths which occur in these simulations, the assumptions of Bowden and Tabor [54] are fulfilled and that the evolution of the friction coefficient with depth for the spherical nanoparticle can be well predicted from purely geometric considerations. This finding agrees with previous NEMD simulations of fixed hard abrasive particles on atomically rough $\alpha$-iron surfaces [33]. The simulated friction data lie slightly above the Bowden-Tabor prediction, particularly at low indentation depths, suggesting another contribution to the friction coefficient. This contribution can be attributed to the Van der Waals force between the opposing slabs and the nanoparticles. Although the indentation depth is greater at each pressure for CND than CNO, the friction coefficient is almost identical at equal indentation depth.

The friction coefficient results obtained in these simulations generally agree well with experimentally observed values for CNO and CND boundary friction experiments using base oils containing CNO and CND. These generally yield friction coefficients of $\approx 0.1$ [12-23], though higher ( $\approx 0.3$ ) values have also been observed [9]. The frictions coefficients obtained experimentally suggest that nanoparticles are indented into the surface because friction coefficients from simulations with no nanoparticle indentation (high coverages and low pressures) are much lower $(<0.05)$. This is supported by HRTEM images of the wear track after sliding which show indentation of the nanoparticles into the surface for both CNO [23] and CND [12]. Comparing experimentally observed friction coefficients $(\approx 0.1)$ to Fig. $9 \mathrm{c}$ suggests a relatively small indentation depth $(\approx 1 \AA)$. These simulations also indicate that indentation depth is very sensitive to nanoparticle coverage and pressure, meaning that indentation depth probably varies significantly around this value within a macroscopic contact, depending on the local conditions.

The relatively small amount of indentation observed experimentally and in these simulations may actually be important to the effective lubrication of hard nanoparticles such as CND and CNO. It has been argued previously that some adhesion with the surface must be necessary in order to maintain an adequate coverage of nanoparticles to maintain separation of contact surfaces [1]. The high specific surface area of CND and CNO, up to $400 \mathrm{~m}^{2} \mathrm{~g}^{-1}$, results in reasonably strong surface-active and adsorption properties [9]. However, given that CND and CNO are nonpolar and only have relatively weak short-ranged Van der Waals interactions with the surface, they probably show less surface activity than, for example, conventional amphiphilic organic friction modifier additives. As a consequence, some indentation into the surfaces could ensure that nanoparticles remain on the surface following compression rather than returning into the oil. This may be crucial in order to maintain an adequate coverage to effectively separate contact surfaces and reduce friction and wear [1].

The $\alpha$-iron slabs used in these simulations are softer than carbon steel and iron oxide, the most relevant surfaces experimentally and industrially, meaning that indentation is more pronounced in these simulations than might be expected experimentally. For example, recent nanoindentation experiments suggested that Fe hardness increases linearly with $\mathrm{C}$ content, by up to an order of magnitude for pure $\mathrm{Fe}_{3} \mathrm{C}$ (cementite) [55]. However, both CND and CNO nanoparticles are harder than carbon steel and hence the high boundary pressure is expected to be accommodated in the same manner, with the slab deforming around the nanoparticle. That said, for a given surface coverage and pressure, the indentation depth of the nanoparticle into the slab will be less in carbon steel than $\alpha$-iron. This in turn will result in reduced wear and a lower friction coefficient, i.e. points on Fig. 9c would be shifted towards the bottom left region of the curve. Nonetheless, assuming that the normal and lateral hardness are equal, the dotted line in Fig. 9c should still predict the friction coefficient of nanoparticles between harder surfaces. Conversely, on softer surfaces [30], such as tribofilms formed from the anti-wear additive zinc dialkyldithiophosphate (ZDDP), the indentation depth may be greater at a given coverage and pressure than suggested by these simulations. In this case, these hard nanoparticles may cause high friction and 
accelerated wear by abrading tribofilms which are reformed, and then abraded again; this is the same mechanism which has been proposed for increased wear from model diesel soot (carbon black) in ZDDP-containing formulations [56]. This contrasts to experimental studies using diesel soot as an additive in the absence of ZDDP, where a significant decrease in friction and wear was observed [57, 58]. These findings suggest that $\mathrm{CNO}$ and particularly CND may lead to increased friction and wear in the presence of ZDDP, a possibility which should certainly be investigated experimentally.

\subsection{Effect of Sliding Velocity}

Under the range of sliding velocities simulated $\left(v_{\mathrm{s}}=5,10\right.$, $20 \mathrm{~m} \mathrm{~s}^{-1}$ ), there was negligible change in the friction and wear behaviour. This is shown for at high pressure $\left(P_{z}=4.0 \mathrm{GPa}\right)$ when the nanoparticles indent into the surface in Fig. 10a, though the same trends are evident at lower pressure $\left(P_{z}=1.0 \mathrm{GPa}\right)$ where the nanoparticles do not indent. However, the average angular velocity of the nanoparticles, which indicates their rolling motion, did change with the sliding velocity (Fig. 10b). In Fig. 10b, a value of zero for the angular velocity indicates that the nanoparticles are sliding, a positive value indicates that the nanoparticles are rolling with the sliding direction, whilst a negative value indicates that the nanoparticles are rolling against it.

There has been much interest in determining whether carbon nanoparticles act as nanoscale ball bearings, i.e. that the rolling motion of the nanoparticles contributes to low friction $[12,23,24]$. At the macroscale, rolling resistance is generally much lower than sliding friction since it provides an additional velocity accommodation mechanism [7, 8]. However, at the atomic scale, experimental studies of $\mathrm{C}_{60}$ fullerene films actually yielded slightly higher friction when molecules were allowed to roll as opposed to when they were fixed in place and forced to slide [7, 8]. Indeed, in these simulations, the angular velocity of the nanoparticles seems to have a minimal effect on the friction coefficient obtained. The nanoparticles undergo short periods of sliding and rolling through the course of the simulations (Fig. 10b); yet, there is minimal change in the friction coefficient (Fig. 10a). Moreover, the friction coefficient at 1.00 coverage, where the nanoparticles do not roll due to the proximity of other nanoparticles, is actually lower than at 0.44 coverage, where rolling occurs. In previous NEMD simulations of CNO between DLC surfaces, lower friction coefficients have been attributed to the rolling motion of the nanoparticles relative to sliding $[23,24]$. However, from the results of these simulations, it is suggested that the differences in the friction coefficients during rolling (low pressure) and sliding (high pressure) actually originated from an increase in adhesive forces, due to surface nanoparticle bond formation, rather than changes to the motion of the nanoparticles. In summary, for the range of conditions simulated here, it seems that rolling motion is not a significant contributor to the low friction achieved by CNO and CND additives, as has been confirmed experimentally for $\mathrm{C}_{60}[7,8]$.

\section{Summary and Conclusions}

In this study, NEMD simulations with accurate atomistic potentials have been successfully utilised to shed light on the lubrication mechanisms of two carbon nanoparticles: CNO and CND. The simulations have analysed the effects of nanoparticle type and coverage, as well as the local pressure and sliding velocity, on the friction coefficient and the indentation (wear) depth.

Both CNO and CND nanoparticles appear to be promising boundary lubricant additives, which yield low friction by maintaining separation of asperities as a result of their high compressive strength and hardness. These simulations indicate that the friction and wear behaviour is highly dependent on the coverage of the nanoparticle and the contact pressure. In order to maximise friction and wear
Fig. 10 a Effect of the sliding velocity, $v_{\mathrm{s}}$, on the friction coefficient. b Average angular velocity of one of the nanoparticles with sliding velocities at different sliding velocities, $v_{\mathrm{s}}=5,10,20 \mathrm{~m} \mathrm{~s}^{-1}$. Data are shown for CNO at $\theta=0.44, P_{z}=4.0 \mathrm{GPa}$. Horizontal dotted lines in b represent angular velocity expected for $100 \%$ rolling (0\% sliding)
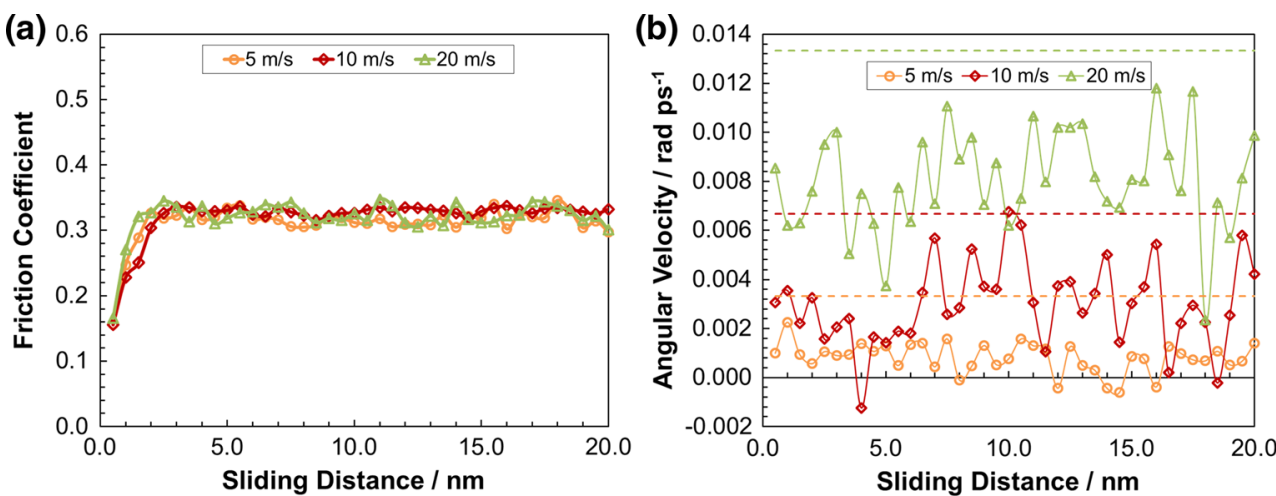
reduction, a high coverage of nanoparticle is required between asperities. This suggests that effective dispersion methods, to avoid agglomeration, are critical to the effective performance of these additives so that they may enter the contact at the requisite coverage.

At high coverage and low pressure, the nanoparticles do not indent into the slabs, leading to a low friction coefficient which arises purely from Van der Waals interactions between the nanoparticles and the slabs. At low coverage and high pressure, which are likely to be encountered in boundary lubrication, nanoparticles indent into, and plough through the slabs during sliding, leading to atomic-scale wear and a much higher friction coefficient. After the onset of indentation, both the indentation (wear) depth of the nanoparticles and the friction coefficient increase linearly with pressure. However, even at the highest pressures and lowest coverages simulated, both types of nanoparticle are able to maintain separation of opposing slabs and reduce friction by approximately $75 \%$ compared to when no nanoparticle is present, in accord with experimental results. The contribution to the friction coefficient due to indentation can be well predicted through purely geometric considerations using an expression developed for macroscopic indentation by Bowden and Tabor. Friction coefficients observed experimentally for $\mathrm{CNO}$ and CND suggest $\approx 1 \AA$ of indentation of the nanoparticles into the surface. This relatively small indentation depth may actually be important to the effective performance of these additives by maintaining a higher coverage between asperities.

As a result of their layered structure, $\mathrm{CNO}$ nanoparticles are softer, and indent less into the $\alpha$-iron surface than CND at the same coverage and pressure. This reduced indentation depth leads to an approximately $25 \%$ reduction in both the indentation (wear) depth and the friction coefficient in these NEMD simulations. This agrees well with differences observed experimentally in boundary friction experiments for $\mathrm{CNO}$ and CND. Therefore, both these simulations and most experimental studies suggest that CNOs are more attractive as boundary lubricant additives.

The indentation depths observed on the $\alpha$-iron slabs used in these simulations are expected to be more pronounced than would occur on the harder carbon steel and iron oxide surfaces more commonly encountered experimentally. However, the pressure-indentation, pressurefriction, and indentation-friction trends observed here are expected to remain the same on these surfaces.

Whilst rolling of the nanoparticles can be observed during these simulations, it does not appear to be critical in reducing the friction coefficient. There is no detectable difference in the friction coefficient in periods where nanoparticles roll or slide, and simulations where more rolling occurs often do not yield a lower friction coefficient. This suggests that the separation of the contact surfaces is the critical factor in the friction reduction of nanoparticles rather than the motion of the nanoparticles themselves.

Acknowledgments JPE acknowledges the financial support of the Engineering and Physical Sciences Research Council (EPSRC) via a Case Conversion studentship and Shell Global Solutions via the University Technology Centre for Fuels and Lubricants at Imperial College London. HAS, CG, and DD thank the EPSRC for support under the Platform Grant EP/G026114/1 and an Established Career Fellowship EP/N025954/1. The authors acknowledge the use of Shell HPC facilities in Houston, USA. JPE would also like to thank Lars Pastewka and Thomas O'Connor as well as the Computational Centre of Expertise group at Shell Technology Centre Bangalore for helpful discussions.

Open Access This article is distributed under the terms of the Creative Commons Attribution 4.0 International License (http://crea tivecommons.org/licenses/by/4.0/), which permits unrestricted use, distribution, and reproduction in any medium, provided you give appropriate credit to the original author(s) and the source, provide a link to the Creative Commons license, and indicate if changes were made.

\section{References}

1. Spikes, H.: Friction modifier additives. Tribol. Lett. 60, 5 (2015)

2. Miura, K., Kamiya, S., Sasaki, N.: C-60 molecular bearings. Phys. Rev. Lett. 90, 4 (2003)

3. Gupta, B.K., Bhushan, B.: Fullerene particles as an additive to liquid lubricants and greases for low-friction and wear. Lubr. Eng. 50, 524-528 (1994)

4. Ginzburg, B.M., Kireenko, O.F., Shepelevskii, A.A., Shibaev, L.A., Tochilnikov, D.G., Leksovskii, A.M.: Thermal and tribological properties of fullerene-containing composite systems. Part 2. Formation of tribo-polymer films during boundary sliding friction in the presence of fullerene C-60. J. Macromol. Sci. B44, 93-115 (2005)

5. Lee, K., Hwang, Y., Cheong, S., Choi, Y., Kwon, L., Lee, J., Kim, S.H.: Understanding the role of nanoparticles in nano-oil lubrication. Tribol. Lett. 35, 127-131 (2009)

6. Ku, B.C., Han, Y.C., Lee, J.E., Lee, J.K., Park, S.H., Hwang, Y.J.: Tribological effects of fullerene (C-60) nanoparticles added in mineral lubricants according to its viscosity. Int. J. Precis. Eng. Manuf. 11, 607-611 (2010)

7. Liang, Q., Tsui, O.K.C., Xu, Y.B., Li, H.N., Xiao, X.D.: Effect of C-60 molecular rotation on nanotribology. Phys. Rev. Lett. 90, 4 (2003)

8. Coffey, T., Krim, J.: C-60 molecular bearings and the phenomenon of nanomapping. Phys. Rev. Lett. 96, 4 (2006)

9. Chou, C.C., Lee, S.H.: Tribological behavior of nanodiamonddispersed lubricants on carbon steels and aluminum alloy. Wear 269, 757-762 (2010)

10. Ugarte, D.: Curling and closure of graphitic networks under electron-beam irradtiation. Nature 359, 707-709 (1992)

11. Hawelek, L., Brodka, A., Tomita, S., Dore, J.C., Honkimaki, V., Burian, A.: Transformation of nano-diamonds to carbon nanoonions studied by X-ray diffraction and molecular dynamics. Diam. Relat. Mater. 20, 1333-1339 (2011)

12. Xu, T., Zhao, J.Z., Xu, K.: The ball-bearing effect of diamond nanoparticles as an oil additive. J. Phys. D Appl. Phys. 29, 2932-2937 (1996) 
13. Peng, D.X., Kang, Y., Hwang, R.M., Shyr, S.S., Chang, Y.P.: Tribological properties of diamond and $\mathrm{SiO}(2)$ nanoparticles added in paraffin. Tribol. Int. 42, 911-917 (2009)

14. Ivanov, M.G., Pavlyshko, S.V., Ivanov, D.M., Petrov, I., Shenderova, O.: Synergistic compositions of colloidal nanodiamond as lubricant-additive. J. Vac. Sci. Technol. B 28, 869-877 (2010)

15. Hsin, Y.L., Chu, H.Y., Jeng, Y.R., Huang, Y.H., Wang, M.H., Chang, C.K.: In situ de-agglomeration and surface functionalization of detonation nanodiamond, with the polymer used as an additive in lubricant oil. J. Mater. Chem. 21, 13213-13222 (2011)

16. Mosleh, M., Shirvani, K.A.: In-situ nanopolishing by nanolubricants for enhanced elastohydrodynamic lubrication. Wear 301, 137-143 (2013)

17. Nunn, N., Mahbooba, Z., Ivanov, M.G., Ivanov, D.M., Brenner, D.W., Shenderova, O.: Tribological properties of polyalphaolefin oil modified with nanocarbon additives. Diam. Relat. Mater. 54, 97-102 (2015)

18. Matsumoto, N., Joly-Pottuz, L., Kinoshita, H., Ohmae, N.: Application of onion-like carbon to micro and nanotribology. Diam. Relat. Mater. 16, 1227-1230 (2007)

19. Joly-Pottuz, L., Matsumoto, N., Kinoshita, H., Vacher, B., Belin, M., Montagnac, G., Martin, J.M., Ohmae, N.: Diamond-derived carbon onions as lubricant additives. Tribol. Int. 41, 69-78 (2008)

20. Joly-Pottuz, L., Vacher, B., Ohmae, N., Martin, J.M., Epicier, T.: Anti-wear and friction reducing mechanisms of carbon nanoonions as lubricant additives. Tribol. Lett. 30, 69-80 (2008)

21. Yao, Y.L., Wang, X.M., Guo, J.J., Yang, X.W., Xu, B.S.: Tribological property of onion-like fullerenes as lubricant additive. Mater. Lett. 62, 2524-2527 (2008)

22. Hunter, C.N., Check, M.H., Hager, C.H., Voevodin, A.A.: Tribological properties of carbon nanopearls synthesized by nickel-catalyzed chemical vapor deposition. Tribol. Lett. 30, 169-176 (2008)

23. Joly-Pottuz, L., Bucholz, E.W., Matsumoto, N., Phillpot, S.R., Sinnott, S.B., Ohmae, N., Martin, J.M.: Friction properties of carbon nano-onions from experiment and computer simulations. Tribol. Lett. 37, 75-81 (2010)

24. Bucholz, E.W., Phillpot, S.R., Sinnott, S.B.: Molecular dynamics investigation of the lubrication mechanism of carbon nanoonions. Comput. Mater. Sci. 54, 91-96 (2012)

25. Matsumoto, N., Mistry, K., Kim, J.-H., Eryilmaz, O., Erdemir, A., Kinoshita, H., Ohmae, N.: Friction reducing properties of onion-like carbon based lubricant under high contact pressure. Tribol. Mater. Surf. Interfaces 6, 116-120 (2012)

26. Jeng, Y.R., Huang, Y.H., Tsai, P.C., Hwang, G.L.: Tribological properties of carbon nanocapsule particles as lubricant additive. J. Tribol. ASME 136, 9 (2014)

27. Persson, B.N.J., Mugele, F.: Squeeze-out and wear: fundamental principles and applications. J. Phys. Condens. Matter 16, R295R355 (2004)

28. Ewen, J.P., Gattinoni, C., Morgan, N., Spikes, H.A., Dini, D.: Nonequilibrium molecular dynamics simulations of organic friction modifiers adsorbed on iron oxide surfaces. Langmuir 32, 4450-4463 (2016)

29. Tevet, O., Von-Huth, P., Popovitz-Biro, R., Rosentsveig, R., Wagner, H.D., Tenne, R.: Friction mechanism of individual multilayered nanoparticles. Proc. Natl. Acad. Sci. USA 108, 19901-19906 (2011)

30. Zhmud, B., Pasalskiy, B.: Nanomaterials in lubricants: an industrial perspective on current research. Lubricants 1, 95-101 (2013)

31. Lahouij, I., Bucholz, E.W., Vacher, B., Sinnott, S.B., Martin, J.M., Dassenoy, F.: Lubrication mechanisms of hollow-core inorganic fullerene-like nanoparticles: coupling experimental and computational works. Nanotechnology 23, 10 (2012)

32. Hu, C.Z., Bai, M.L., Lv, J.Z., Kou, Z.H., Li, X.J.: Molecular dynamics simulation on the tribology properties of two hard nanoparticles (diamond and silicon dioxide) confined by two iron blocks. Tribol. Int. 90, 297-305 (2015)

33. Eder, S.J., Feldbauer, G., Bianchi, D., Cihak-Bayr, U., Betz, G., Vernes, A.: Applicability of macroscopic wear and friction laws on the atomic length scale. Phys. Rev. Lett. 115, 025502 (2015)

34. Plimpton, S.: Fast parallel algorithms for short-range moleculardynamics. J. Comput. Phys. 117, 1-19 (1995)

35. Stuart, S.J., Tutein, A.B., Harrison, J.A.: A reactive potential for hydrocarbons with intermolecular interactions. J. Chem. Phys. 112, 6472-6486 (2000)

36. O'Connor, T.C., Andzelm, J., Robbins, M.O.: AIREBO-M: a reactive model for hydrocarbons at extreme pressures. J. Chem. Phys. 142, 9 (2015)

37. Brenner, D.W., Shenderova, O.A., Harrison, J.A., Stuart, S.J., Ni, B., Sinnott, S.B.: A second-generation reactive empirical bond order (REBO) potential energy expression for hydrocarbons. J. Phys. Condens. Matter 14, 783-802 (2002)

38. Mendelev, M.I., Han, S., Srolovitz, D.J., Ackland, G.J., Sun, D.Y., Asta, M.: Development of new interatomic potentials appropriate for crystalline and liquid iron. Philos. Mag. 83, 3977-3994 (2003)

39. Zheng, X., Zhu, H.T., Tieu, A.K., Kosasih, B.: Roughness and lubricant effect on 3D atomic asperity contact. Tribol. Lett. 53, 215-223 (2014)

40. Spijker, P., Anciaux, G., Molinari, J.F.: The effect of loading on surface roughness at the atomistic level. Comput. Mech. 50, 273-283 (2012)

41. Banerjee, S., Naha, S., Puri, I.K.: Molecular simulation of the carbon nanotube growth mode during catalytic synthesis. Appl. Phys. Lett. 92, 3 (2008)

42. Gao, Y., Ruestes, C.J., Urbassek, H.M.: Nanoindentation and nanoscratching of iron: atomistic simulation of dislocation generation and reactions. Comput. Mater. Sci. 90, 232-240 (2014)

43. Gao, Y., Brodyanski, A., Kopnarski, M., Urbassek, H.M.: Nanoscratching of iron: a molecular dynamics study of the influence of surface orientation and scratching direction. Comput. Mater. Sci. 103, 77-89 (2015)

44. Humphrey, W., Dalke, A., Schulten, K.: VMD: visual molecular dynamics. J. Mol. Graph. Model 14, 33-38 (1996)

45. Schneider, T., Stoll, E.: Molecular-dynamics study of a threedimensional one-component model for distortive phase-transitions. Phys. Rev. B 17, 1302-1322 (1978)

46. Bernardi, S., Todd, B.D., Searles, D.J.: Thermostating highly confined fluids. J. Chem. Phys. 132, 10 (2010)

47. Hu, C.Z., Bai, M.L., Lv, J.Z., Liu, H., Li, X.J.: Molecular dynamics investigation of the effect of copper nanoparticle on the solid contact between friction surfaces. Appl. Surf. Sci. 321, 302-309 (2014)

48. Abouei, V., Saghafian, H., Kheirandish, S.: Effect of microstructure on the oxidative wear behavior of plain carbon steel. Wear 262, 1225-1231 (2007)

49. Lee, W.G., Cho, K.H., Jang, H.: Molecular dynamics simulation of rolling friction using nanosize spheres. Tribol. Lett. 33, 37-43 (2009)

50. Guo, J.J., Liu, G.H., Wang, X.M., Fujita, T., Xu, B.S., Chen, M.W.: High-pressure Raman spectroscopy of carbon onions and nanocapsules. Appl. Phys. Lett. 95, 3 (2009)

51. Zhang, W.W., Yao, M.G., Fan, X.H., Zhao, S.J., Chen, S.L., Gong, C., Yuan, Y., Liu, R., Liu, B.B.: Pressure-induced transformations of onion-like carbon nanospheres up to $48 \mathrm{GPa}$. J. Chem. Phys. 142, 6 (2015)

52. Gattinoni, C., Heyes, D.M., Lorenz, C.D., Dini, D.: Traction and nonequilibrium phase behavior of confined sheared liquids at high pressure. Phys. Rev. E 88, 10 (2013)

53. Lu, C., Gao, Y., Michal, G., Deng, G.Y., Huynh, N.N., Zhu, H.T., Liu, X.H., Tieu, A.K.: Experiment and molecular dynamics simulation of nanoindentation of body centered cubic iron. J. Nanosci. Nanotechnol. 9, 7307-7313 (2009) 
54. Bowden, F., Tabor, D.: Friction, lubrication and wear: a survey of work during the last decade. Br. J. Appl. Phys. 17, 1521-1544 (1966)

55. Zheng, B., Huang, Z., Xing, J., Wang, Y., Jian, Y., Xiao, Y., Fan, X.: Three-body abrasive behavior of cementite-iron composite with different cementite volume fractions. Tribol. Lett. 62, 1-11 (2016)

56. Olomolehin, Y., Kapadia, R., Spikes, H.: Antagonistic interaction of antiwear additives and carbon black. Tribol. Lett. 37, 49-58 (2010)
57. Bhowmick, H., Majumdar, S.K., Biswas, S.K.: Influence of physical structure and chemistry of diesel soot suspended in hexadecane on lubrication of steel-on-steel contact. Wear 300, 180-188 (2013)

58. Zhang, Z.C., Cai, Z.B., Peng, J.F., Zhu, M.H.: Comparison of the tribology performance of nano-diesel soot and graphite particles as lubricant additives. J. Phys. D Appl. Phys. 49, 45304 (2016) 